\title{
NUEVA LOCALIDAD EN CHILE PARA MENODORA LINOIDES PHIL. (OLEACEAE), ESPECIE CONSIDERADA EXTINTA EN EL SIGLO XX
}

\section{NEW LOCALITY IN CHILE FOR MENODORA LINOIDES PHIL. (OLEACEAE), SPECIES CONSIDERED EXTINT IN THE 2OTH CENTURY}

\author{
Mélica Muñoz-Schick ${ }^{1}$,Andrés Moreira-Muñoz ${ }^{2} \&$ Pedro León-Lobos ${ }^{3}$ \\ ${ }^{1}$ Museo Nacional de Historia Natural, Casilla 787, Santiago, Chile, mmunoz@mnhn.cl \\ ${ }^{2}$ Institut für Geographie, Universität Erlangen-Nürnberg, Koch Str.4/4, D-91054, Alemania \\ ${ }^{3}$ Banco Base de Semillas, Instituto de Investigaciones Agropecuarias, INIA, Casilla 73, Vicuña, Chile.
}

\section{ABSTRACT}

An area in the Andes of the Valparaíso Region is indicated as a new locality for the species Menodora linoides Phil., an endemic and unique representative of the Oleaceae family in Chile. The plant described in 1863, was considered extinct in the $20^{\text {th }}$ century, but has been collected twice in the present century.

\section{INTRODUCCION}

R.A. Philippi describe en 1863 la especie Menodora linoides, que constituye así la única especie de la familia Oleaceae representada en Chile. La planta fue recolectada por uno de sus colaboradores, L. Landbeck, entre Concumén y Chincolco (V Región de Valparaíso) en el mes de enero, pero después de ello no volvió a ser encontrada en la naturaleza. Por tal motivo fue catalogada como planta Rara en el Libro Rojo de CONAF (Benoit 1989), basándose en la publicación de Muñoz (1973), Chile: Plantas en Extinción. Posteriormente Squeo et al. (2001) la consideraron extinta para la Región de Coquimbo, ya que existe un Concumén en la provincia de Choapa, pero en los muestreos realizados no la encontraron.

El género Menodora Humb. et Bonpl., con cerca de 17 especies, tiene una distribución disyunta en Norte y Sudamérica y además está presente en Africa del Sur (Figura 1A). En ambos continentes, algunas especies penetran en los trópicos, pero está ausente en Centroamérica y norte de Sudamérica.

En cuanto a nuestros países limítrofes, el género Menodora está representado en Argentina, con 5 especies, 2 de ellas en la región del monte en la
Patagonia (Correa 1999) y en Bolivia con 2 especies (Meyer 1957). No está representado en Perú (Brako y Zarucchi 1993).

Se ha indicado además $M$. decemfida (Gillies) A.Gray, para Chile, quizás por la citación errónea que le agrega Steyermark (1932) a la localidad tipo: valles de los andes de Mendoza, "Chile central", lo que indica como erróneo Meyer (1957), pero nuevamente mal citada para nuestro país en el Catálogo de Argentina (Zuloaga y Morrone 1999).

Al parecer la especie chilena Menodora linoides se encuentra cercanamente emparentada con $M$. integrifolia Steud., especie de amplia distribución en Argentina y regiones limítrofes de Bolivia, $\mathrm{Pa}$ raguay, Brasil y Uruguay. Menodora integrifolia forma el clado hermano de las especies norteamericanas, de forma que la posición de $M$. linoides resulta muy interesante para resolver la filogenia. Estudios moleculares están siendo llevados a cabo para dar respuesta a estas interrogantes (T. Chumley, com. pers.).

\section{Nuevas Colectas}

Menodora linoides estuvo sin ser recolectada desde su descripción en 1863, hasta el año 2004, en que fue colectada en el portezuelo de Pedernales, zona límite entre la V Región y la IV Región 
$32^{\circ} 03^{\prime} \mathrm{S}, 70^{\circ} 49^{\prime} \mathrm{W}$, entre 1650 y $1800 \mathrm{msnm}$ (Faúndez et al. 2005) (SGO 152232).

Con motivo de un proyecto de ordenación y actualización del Herbario SGO, se revisó en el mes de marzo 2006 una colección de A. Moreira, que se realizara entre las localidades de Catemu y Cabildo, en el marco del proyecto Conservación ex situ de Plantas Endémicas, Vulnerables y En Peligro de Extinción, de las zonas desérticas y mediterráneas de Chile, ejecutado por INIA con apoyo del Millenium Seed Bank Project del Royal Botanic Gardens Kew.

En dicha colección se encontró un segundo ejemplar de $M$. linoides recolectado entre la Mina Los Mantos y Las Majadas, 32³8' S-70 52'W, a 1240 msnm (SGO 147940), en el camino interior entre Catemu y cerro Negro, provincia de Petorca, región de Valparaíso (Figura 1B). La recolección fue realizada a fines de diciembre del año 2001.

A mediados de marzo de 2006 se procedió a ir nuevamente al lugar para realizar observaciones y mediciones en terreno. (M. Muñoz n 4805 y n4805 A, 19/03/2006, SGO 152771 y 153172).

La población es pequeña, de unos $5.150 \mathrm{~m}^{2}$ hacia el norte y sur del camino, donde termina en una pequeña hondonada húmeda con presencia de Escallonia illinita C.Presl.

En una extensión de $100 \mathrm{~m}^{2}$ se contaron 75 plantas como especie dominante y un $20 \%$ de cobertura, entre suelo tipo maicillo desnudo y pedregoso. Entre la población aparecían algunos arbustos bajos de Schinus polygama (Cav.) Cabrera, y Baccharis linearis (Ruiz et Pav.)Pers., arbolitos de Acacia caven (Molina) Molina y un arbusto alto de Trevoa quinquenervia (Gillies et Hook.) I.M.Johnst. (Figura 2).

Philippi (1863) no vio frutos ni tampoco Muñoz (1973), quien se basó en los ejemplares tipo de Philippi (SGO 53888 y 38535) para presentar una lámina detallada de la especie; Faúndez et al. (2005) describen la especie en flor. Como nuestro encuentro fue en fruto podemos complementar la descripción como sigue:

Subarbusto de tallo grueso, ramificado desde la base, intrincado, de color verde oscuro, formando grupos de 10-60 cm de diámetro, por 5-20 (-35) cm de alto. Hojas opuestas, sésiles, de bordes enteros, linear- oblongas, a algo elípticas, de 5-10 (-19) x 1,5-3(-4) mm, ápice redondeado hasta apiculado, nervio notorio por el envés, puberulentas, al igual que los tallos. Flores solitarias en el ápice de las ramas, hermafroditas, cáliz turbinado, puberulento y glandulífero, tubo de $3 \mathrm{~mm}$ de largo y 5 divisiones lineares de $4 \mathrm{~mm}$ de largo, con el nervio medio también notorio; corola infundibuliforme, color amarillo-oro, a veces rojiza por fuera, glabra, de tubo corto, ciliado en el interior, que se despliega en 5 partes ovadas, de 10-12 (-15) x 4-5 (-6) mm; ovario súpero, bilocular, estilo largo terminado en estigma capitado; estambres 2, insertos en el tubo corolar, filamentos el doble del largo de las anteras que son lineares y dorsifijas, de 3-3,5 mm de largo. Cápsula acorazonada, erecta, de 6-7 x 6-7 mm, rodeada por el cáliz ensanchado, dehiscente y circuncisa, de color violáceo y algo pegajosa inmadura, y de color café, seca y casi papirárea, cuando madura; lóculos 1-2, semillas 1-2 por lóculo, obovoides, de 4-5 x 2,5-3,5 mm, de color marrón, con testa foveolada, brillante, la cara interna de las semillas pares en cada lóculo es plana o con un pequeño reborde, externamente son redondeadas, las que al caer el opérculo de la cápsula, donde queda la base con las semillas, caen con cualquier movimiento (Figuras 3A-E).

Por las características del fruto, erecto y con 4 óvulos en cada celda, pertenecería al subgénero Eumenodora (Steyermark 1932).

Las plantas de Los Mantos - Las Majadas se encontraron bastante ramoneadas por el ganado y no se observaron plántulas. El significado del nombre del género, del griego, menos = "agradable", y doron = "regalo, ofrenda, vegetal que da fuerza a los animales" ya sugiere su uso alimenticio por el ganado, ya que las especies argentinas también se presentan muy ramoneadas por chivos y ovejas (Correa 1999).

Tanto el portezuelo de Pedernales como la localidad entre Mina Los Mantos y Las Majadas no se encuentran insertas en el Sistema Nacional de Areas Silvestres Protegidas del Estado (SNASPE), por lo que la especie se encuentra en serio Peligro de Extinción.

Por lo pronto la localidad Los Mantos-Las Majadas, la más austral señalada hasta ahora para $M$. linoides, es un aporte al conocimiento de esta especie que vuelve a ser colectada luego de más de 140 años. Ahora se presenta el desafío de cultivar la especie ex situ y estudiar su biología tanto en terreno como bajo cultivo. Para tal efecto se entregaron semillas al Jardín Botánico Chagual, al Jardín Botánico Nacional, al Jardín Botánico de Talca y para su conservación a futuro, al Banco Base de Semillas de INIA en Vicuña. 


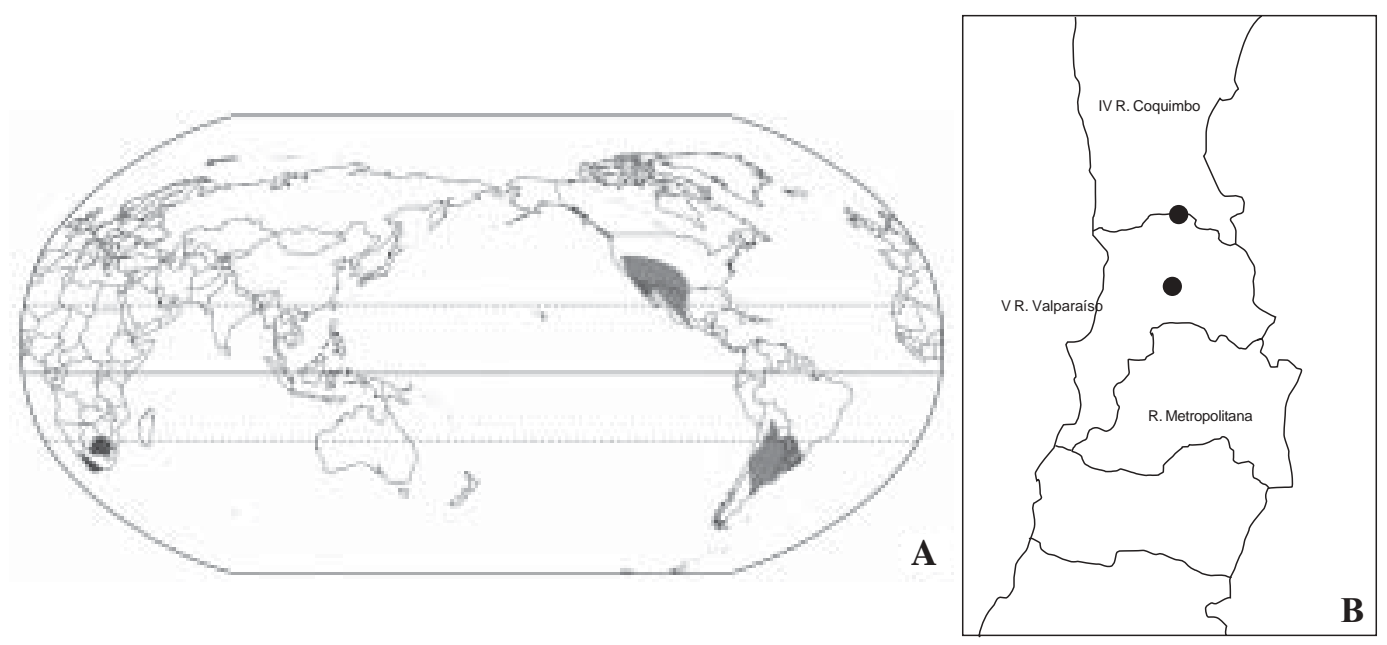

Figura 1. A. Distribución mundial del género Menodora. B. Puntos de localización de las poblaciones de Menodora linoides.

FIGURE 1. A. Global distribution of the genus Menodora. B. Localization points of the Menodora linoides populations.

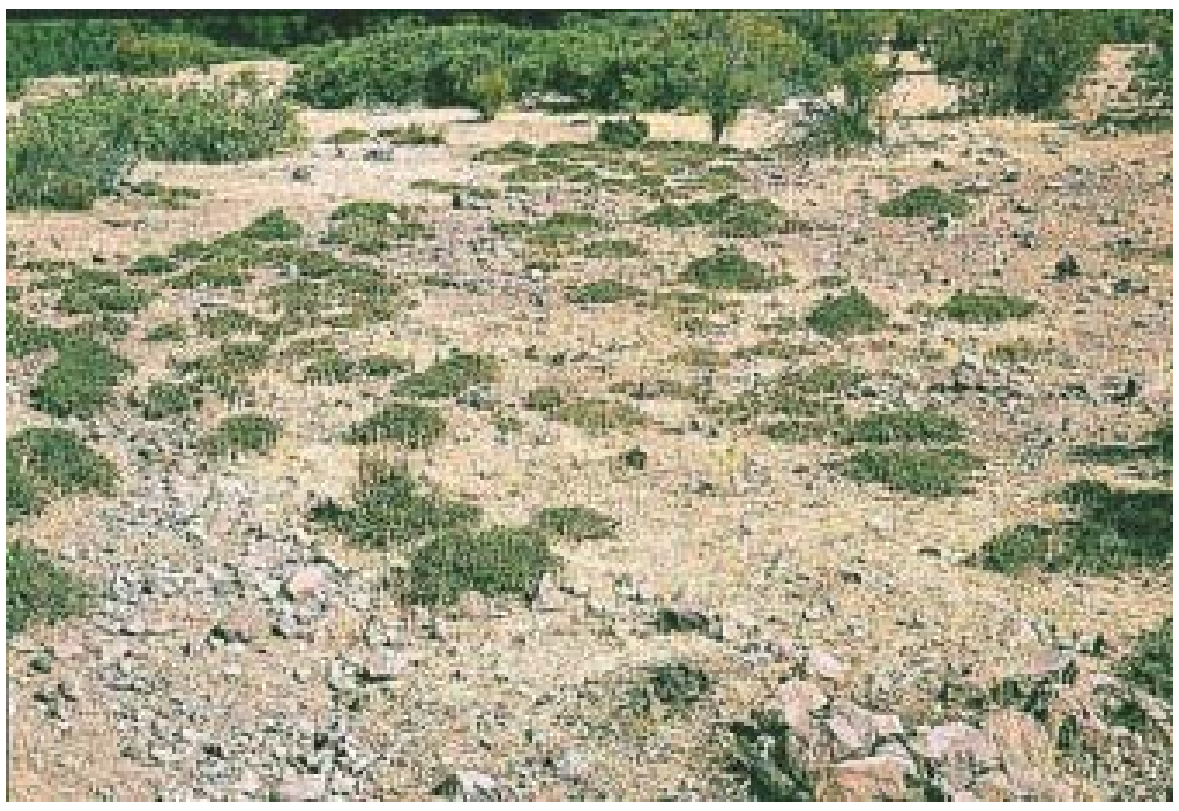

FIGURA 2. Primer plano con Menodora, atrás Schinus y Baccharis (Fotografía de Sergio Moreira E.).

FIGURE 2. In front Menodora, on the back Schinus and Baccharis (Photograph by Sergio Moreira E.). 

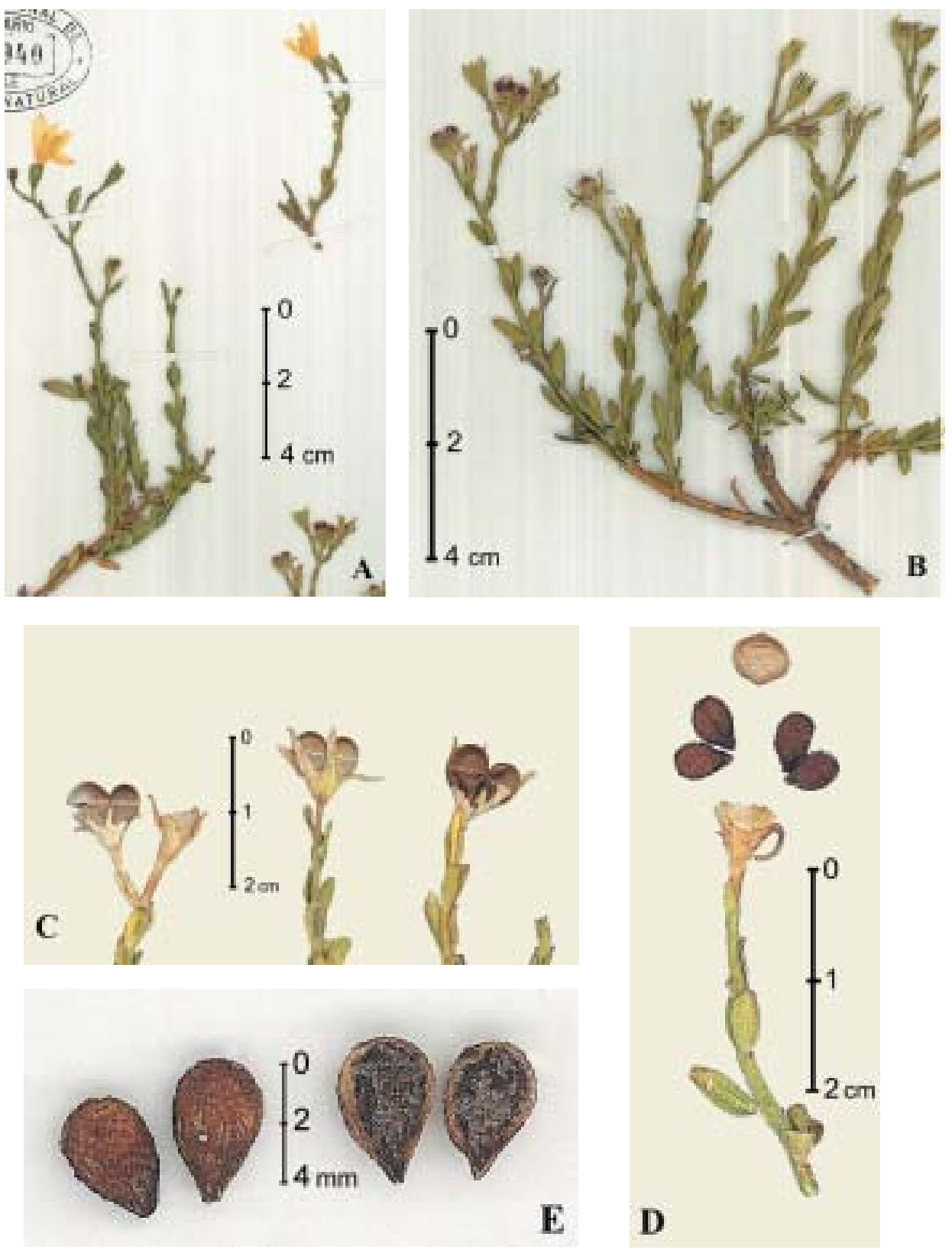

Figura 3. A Hojas, flores y frutos. B. Rama con frutos inmaduros.C. Frutos maduros circuncisos. D. Cápsula abierta que contiene cuatro semillas. E. Semillas de una cápsula, mostrando testa foveolada, izq. cara dorsal, der. cara ventral (A, B: SGO 147940; C, D, E: SGO 152771).

Figure 3. A. Leaves, flowers and fruits. B. Stem with inmature fruits C. Circumscissile mature fruits. D. Open capsule showing four seeds. E. Seeds of one capsule showing the foveolate testa, dorsal face on the left, ventral face on the right (A, B: SGO 147940; C, D, E: SGO 152771). 


\section{AGRADECIMIENTOS}

Se agradece a Sergio Moreira por su constante apoyo y las fotos y mediciones en terreno. A María Teresa Eyzaguirre Philippi, por su entusiasmo en el reencuentro de una especie descrita por su famoso antecesor. También a Francisco Casado por el apoyo logístico de terreno, tanto en la colecta de semillas como en la provisión alimenticia en el proyecto INIA-Kew.

\section{BIBLIOGRAFIA}

BEnOIT, I. (ed.). 1989. Libro Rojo de la flora terrestre de Chile. CONAF 157 pp., 1 mapa.

Brako, L. \& J.L. Zarucchi. 1993. Catálogo de las Angiospermas y Gimnospermas del Perú. Monograph Systematic Botany from the Missouri Botanical Garden 45: 1-1.286.

Correa, M.N. 1999. Oleaceae en Correa, M.N. (ed.) Flora Patagónica VIII, parte VI. Colección Científica del INTA, Bs.As. Argentina. 536 pp.

Faúndez, L., B. Larraín \& G. Girón. 2005.
Redescubrimiento de Menodora linoides Phil. (Oleaceae), una especie considerada como "extinta", en la precordillera de Petorca-Choapa (Regiones de Coquimbo y Valparaíso), Chile. Chloris Chilensis Año $8 \mathrm{~N}^{\mathrm{o}}$ 2. URL: http:// www.chlorischile.cl

Meyer, T. 1957. Las especies de Menodora (Oleaceae) de Argentina, Bolivia, Paraguay y Uruguay. Lilloa 28: 209- 245, 7 figs.

Muñoz, C. 1973. Chile: Plantas en extinción. Editorial Universitaria, Santiago, Chile. 248 pp.

PhILIPPI, R.A. 1863. Descripción de algunas plantas nuevas chilenas. Anales de la Universidad de Chile 23: $376-387$.

Squeo, F., Arancio, G. \& J. Gutiérrez (eds.). 2001. Libro Rojo de la Flora Nativa y de los Sitios Prioritarios para su Conservación: Región de Coquimbo. Ediciones Universidad de La Serena, Chile. 372 pp.

Steyermark, J.A. 1932. A revision of the genus Menodora. Ann. Missouri Botanical Garden 19: 87-160.

Zuloaga F. \& O. Morrone. 1999. Catálogo de las Plantas Vasculares de la República Argentina II, Monographs of Systematic Botany from the Missouri Botanical Garden 74:1-1.269. 